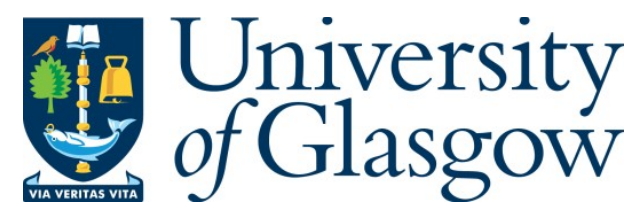

Courtial, J., Gray, N., O'Donnell, R., MacSporran, R., Oxburgh, S., Hendry, M., and Cowie, E. N. (2016) Photography at relativistic speeds. Proceedings of SPIE, 9948, 994808. (doi:10.1117/12.2237850).

Copyright 2016 Society of Photo-Optical Instrumentation Engineers. One print or electronic copy may be made for personal use only. Systematic reproduction and distribution, duplication of any material in this paper for a fee or for commercial purposes, or modification of the content of the paper are prohibited.

http://eprints.gla.ac.uk/129867/

Deposited on: 10 October 2016

Enlighten - Research publications by members of the University of Glasgow http://eprints.gla.ac.uk 


\title{
Photography at relativistic speeds
}

\author{
Johannes Courtial, Norman Gray, Ruaridh O’Donnell, Ross MacSporran, Stephen Oxburgh, \\ Martin Hendry, and Euan N. Cowie
}

School of Physics \& Astronomy, University of Glasgow, Glasgow G12 8QQ, Scotland

\begin{abstract}
In a photo taken with a camera moving at relativistic speed, the world appears distorted. That much has long been clear, but the details of the distortion were slow to emerge correctly. We recently added relativistic raytracing capability to our custom raytracer, Dr TIM, resulting in unique combinations of capabilities. Here we discuss a few observations. In particular, photos can be sharp only if the shutter is placed correctly. A hypothetical window that changes light-ray direction like a change of inertial frame, when combined with suitable shutter placement, can correct for all relativistic-aberration effects.
\end{abstract}

Keywords: Lorentz transformation; relativistic aberration; shutter model

\section{INTRODUCTION}

Relativistic aberration, the apparent distortion of fast-moving objects due to the effects of Special Relativity, started to be discussed even before Special Relativity itself had been formulated: FitzGerald suggested the Lorentz-FitzGerald contraction (or Lorentz contraction) ${ }^{1}$ as an explanation for the Michelson-Morley experiment. $^{2}$ Einstein then discussed it in the same paper in which he formulated Special Relativity. ${ }^{3}$

However, it took a number of years for researchers to realise that the Lorentz-FitzGerald contraction does not correctly describe what fast-moving objects look like. Lampa ${ }^{4}$ took into account the time when the relevant light rays have to leave a moving object in order to arrive simultaneously at the observer. Seen in those light rays, fast-moving objects look different. In 1959, Penrose $^{5}$ and Terrell ${ }^{6}$ independently noted that fast-moving objects would not appear contracted. In the particular cases they studied, the objects would appear rotated, hence this effect is now known as Penrose-Terrell rotation. It quickly became clear that this is not the full story either: Boas ${ }^{7}$ noticed that straight lines can appear curved when fast moving.

Raytracing as a way of visualising fast-moving objects was first used in $1995 .{ }^{8}$ The timing when the light rays arrived at the fast-moving virtual camera was determined by the so-called shutter model, which determines the placement of the shutter within the camera and the time (usually an instant) in which it is open. Noteworthy extensions of this approach include an interactive museum exhibit that is controlled by the observer riding an exercise bike, ${ }^{9}$ and the freely available Real Time Relativity ${ }^{10}$ which uses the graphics card to achieve real-time, interactive, simulation of relativistic speeds.

We became interested in relativistic raytracing when we wanted to use our raytracer TIM $^{11}$ to test the hypothesis that a particular micro-structured window ${ }^{12}$ can make a resting scene seen through it distorted as if it was moving at relativistic speed. This required adding to TIM relativistic raytracing capabilities. The new version, Dr TIM, ${ }^{13}$ allowed us to show that the original hypothesis was wrong. ${ }^{14}$

Here we use Dr TIM's new capabilities to visualise a number of relativistic-aberration effects. We model photos taken with a very simple, and highly idealised, camera whose lens is an ideal thin lens that can image any plane (and indeed more general surfaces) onto the detector, stigmatically. The aperture is defined by the radius of the circular lens. We simply show the images here, with a brief description but without explanation, which will be provided in a separate paper.

This paper is structured as follows. In section 2 we review our implementation of non-relativistic and relativistic raytracing. Then, in section 3, we show the effect of the shutter model on the distortion, and in section 4 we demonstrate the ability of a hypothetical light-ray-direction-changing Lorentz window to correct for the effects of relativistic aberration. Section 5 contains concluding remarks.

Further author information: (Send correspondence to J.C.)

J.C.: E-mail: johannes.courtial@glasgow.ac.uk, Telephone: +44 (0)141 3306429

Novel Optical Systems Design and Optimization XIX, edited by Arthur J. Davis, Cornelius F. Hahlweg,

Joseph R. Mulley, Proc. of SPIE Vol. 9948, 994808 - (C) 2016 SPIE

CCC code: $0277-786 \mathrm{X} / 16 / \$ 18 \cdot$ doi: $10.1117 / 12.2237850$

Proc. of SPIE Vol. $9948994808-1$ 


\section{RELATIVISTIC RAYTRACING}

Consider the ray optics of a camera taking a photo of a scene. Physically, light rays leave light sources and then interact with the scene. Of all these light rays, only a tiny fraction happens to enter the camera and contribute to the photo. For this reason, it is not normally efficient to trace light rays forwards, i.e. in chronological order, starting from the light source. Instead, light rays are usually traced backwards, i.e. in reverse chronological order, starting at the camera. In this way, only light rays that contribute to the photos are being traced.

Different rays are being traced independently. Ray tracing starts at a point, Q, where the ray intersects a scene object, initially a point on the camera lens. To trace a ray to the previous point, $\mathrm{P}$, where it interacts with the scene, raytracing programs calculate the nearest intersection point of the straight line on which the ray travelled before hitting a scene object at $\mathrm{Q}$ and all scene objects. The interaction between the ray and the scene object is then modelled (e.g. absorption, reflection, refraction), and if necessary the ray is being traced further.

If the scene is moving, then the movement of the objects relative to the rest frame of the camera has to be taken into account. The easiest way to do this is to transform the ray from the camera's rest frame into the rest frame of the scene. We assume that the camera is moving relative to the scene with constant velocity $\boldsymbol{v}=\boldsymbol{\beta} c$, where $c$ is the speed of light, and both the camera's rest frame and the scene's rest frame are inertial frames. The transformation from the camera frame into the scene frame can then be performed by Lorentz-transforming the ray. Note that it is very easy to simulate camera filters that move with the camera and are close to it by first tracing the ray through the "camera-frame scene" consisting of all these filters, and only once the part of the ray is reached that has not previously intersected with the camera-frame scene is the ray transformed into the scene frame. If the ray passes through position $\boldsymbol{x}$ at time $t$ in the camera frame, then in the scene frame this event occurs at position ${ }^{8}$

$$
\boldsymbol{x}^{\prime}=\boldsymbol{x}+(\gamma-1) \frac{(\boldsymbol{\beta} \cdot \boldsymbol{x}) \boldsymbol{\beta}}{\beta^{2}}+\gamma \boldsymbol{\beta} c t
$$

where $\gamma=1 / \sqrt{1-\beta^{2}}$. A normalised ray direction $\hat{\boldsymbol{d}}$ in the camera frame becomes a (not necessarily normalised) scene-frame direction ${ }^{14}$

$$
\boldsymbol{d}^{\prime}=\hat{\boldsymbol{d}}+(\gamma-1)(\hat{\boldsymbol{\beta}} \cdot \hat{\boldsymbol{d}}) \hat{\boldsymbol{\beta}}+\gamma \boldsymbol{\beta},
$$

where $\hat{\boldsymbol{\beta}}=\boldsymbol{\beta} / \beta$ is a unit vector in the direction of $\boldsymbol{\beta}$.

Fig. 1 shows examples of photos of the same scene, one taken with a simulated camera at rest, the other taken with a camera moving with a velocity close to $c$.

\section{EFFECT OF THE SHUTTER MODEL}

The shutter model, that is, the placement of the shutter surface and the time the shutter opens, affects the distortion of the scene. Fig. 1(b) was calculated for the Aperture-plane shutter model, in which the shutter is placed in the plane of the aperture (and the lens). The effect of choosing a different shutter model can be seen in Fig. 2, which was calculated for the detector-plane shutter model, in which the shutter is placed in the plane of the detector and the (ideal) lens delays light rays such that all light rays that travel from an object position to the corresponding image position via the lens take the same time to do so. Trivially, comparison of Figs 2(a) and (b), which were simulated for different shutter-opening times, shows differences entirely due to the different camera positions that corresponding to taking pictures at different times with a moving camera. More importantly, comparison of Figs 1(b) and 2(a) reveals subtle differences in the distortions.

More importantly still, Fig. 3(a) reveals that a camera that uses the detector-plane shutter model is able to focus sharply on moving objects. In fact, a full analysis shows that such a camera retains its ability to image moving objects stigmatically, provided it has the ability to image objects at rest stigmatically, which is of course an idealisation. It can also be shown that a camera that uses the aperture-plane shutter model does not retain this stigmatic-imaging ability, and the image shown in Fig. 3(b) is consistent with this. 
a

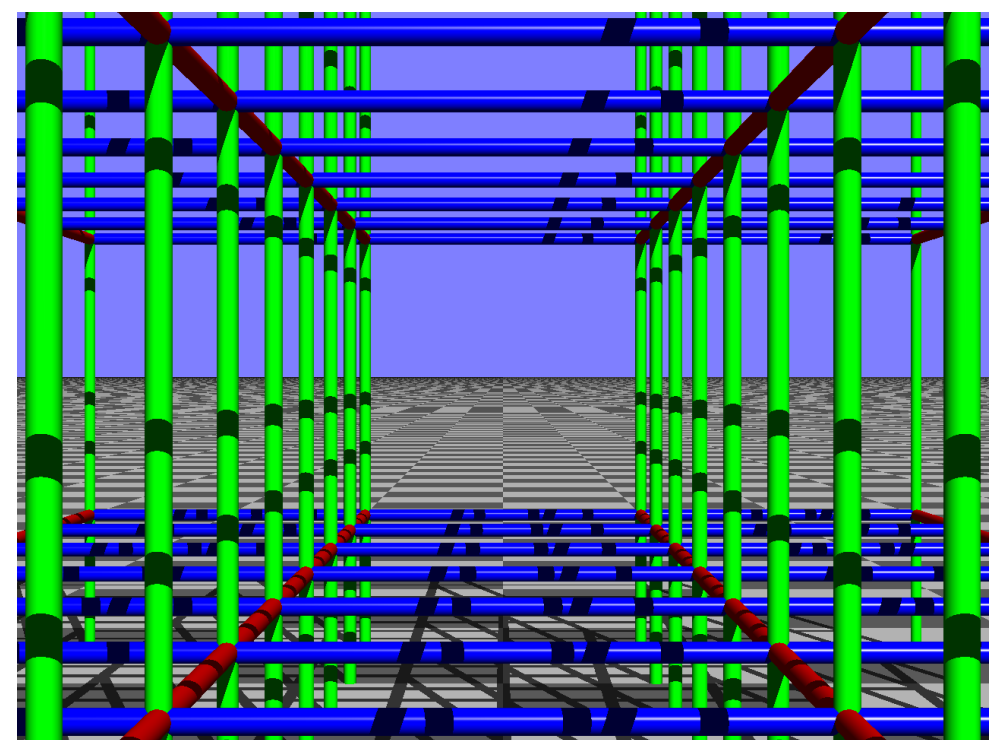

b

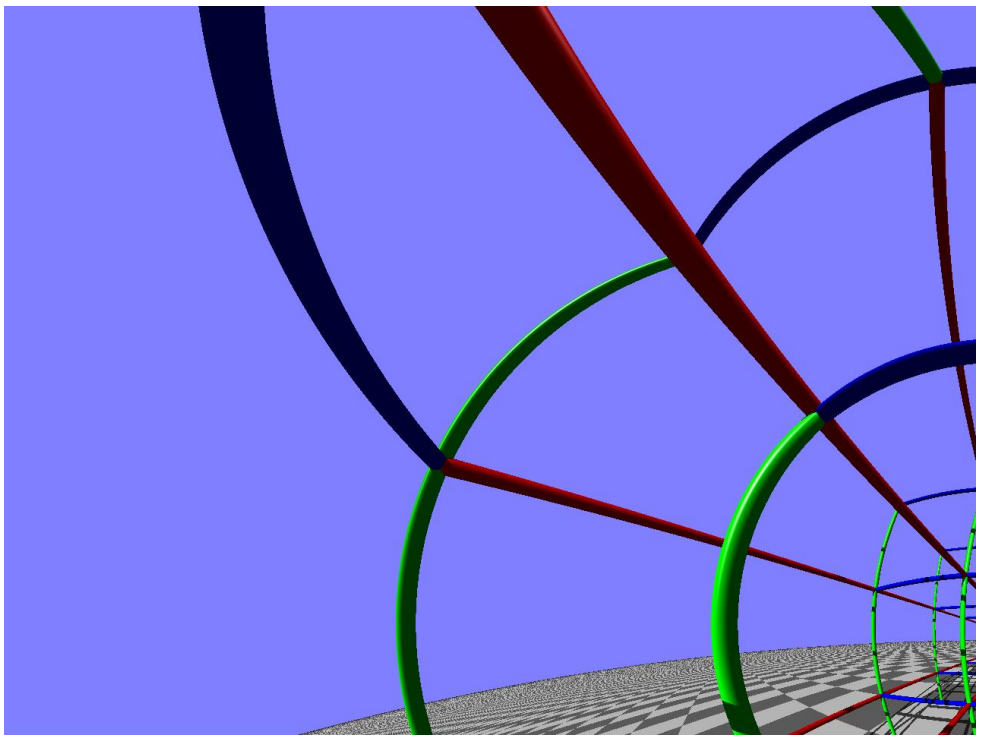

Figure 1. Example of a scene rendered for a camera at rest in the scene (a) and a camera moving through the scene with velocity $\boldsymbol{v}=(0.2,-0.1,0.97)^{\top} c$ in a left-handed coordinate system in which the positive $x$ direction is to the right, the positive $y$ direction upwards, and the positive $z$ direction into the image (b). Both images were rendered using Dr TIM.

\section{CORRECTING RELATIVISTIC ABERRATION}

A camera that uses the aperture-plane shutter model does not retain the ability to image moving objects stigmatically, but it can be corrected for relativistic aberration. In section 2 we discussed the transformation of the light ray from the camera frame to the scene frame during the raytracing process. by placing a Lorentz window, a window that changes the direction of transmitted light rays according to Eqn (2), directly in front of the lens, the light-ray-direction change due to the Lorentz transformation can be exactly cancelled.

Fig. 4 shows an example of a photo simulated for precisely the same parameters as the photo shown in Fig. 1(b), but with a suitable Lorentz window in front of the camera. The resulting, relativistic-aberration-corrected, photo then looks like a photo taken with a camera at rest with respect to the scene. 


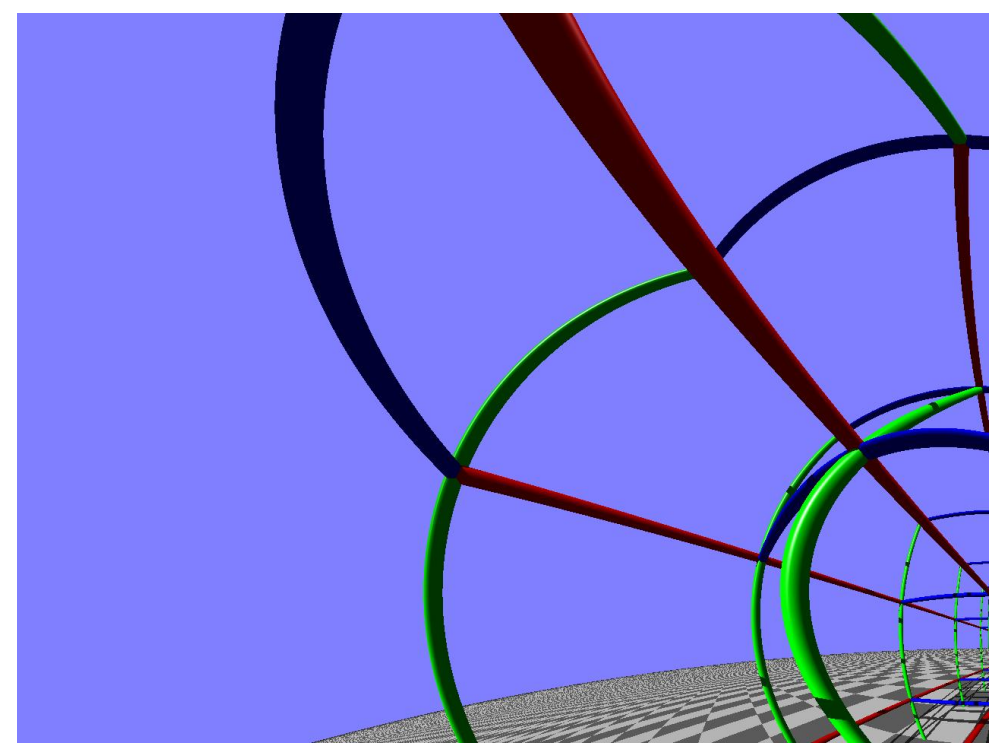

b

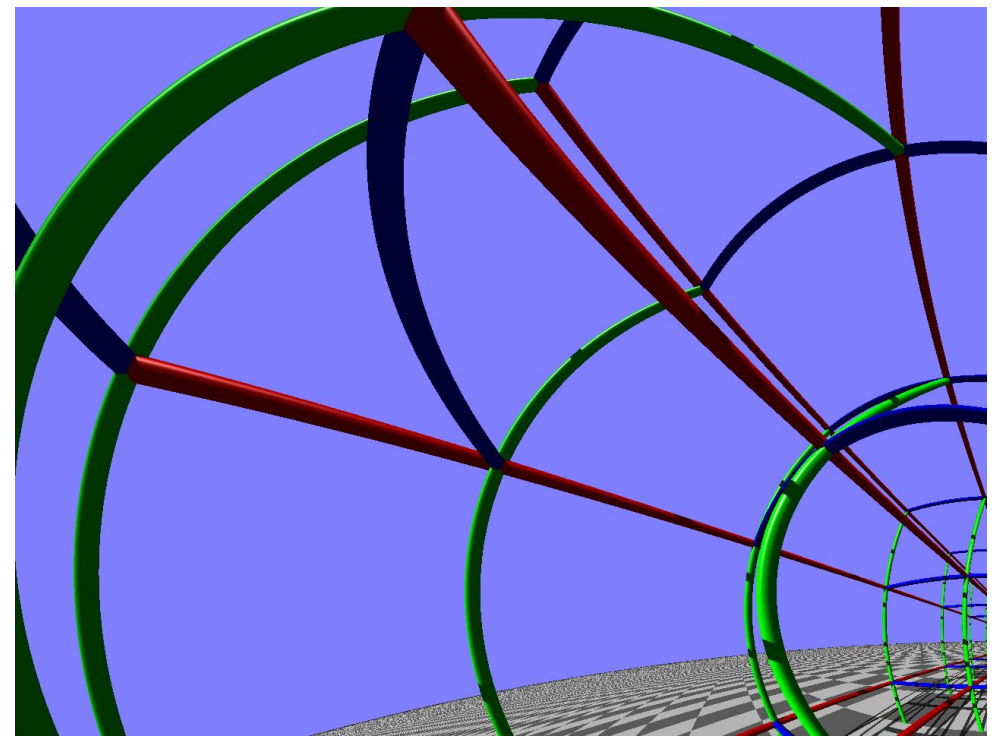

Figure 2. Simulated photos taken with a camera that uses the detector-plane shutter model. In (a), the shutter opens at $t=1.0$, in (b) at $t=1.1$. The scene and other parameters are identical to those used in Fig. 1.

\section{CONCLUSIONS}

We have shown here raytracing simulations that demonstrate a number of new effects related to relativistic aberration. Specifically, the placement of the shutter matters for the sharpness of the resulting photos, and it is possible to correct for the relativistic aberration with a suitable combination of shutter model and light-raydirection-changing window in front of the lens.

The focussing effect is relevant only in unusual circumstances, namely when the object to be photographed is very close to the camera and moving at significant speed.

\section{ACKNOWLEDGMENTS}

This work was supported by the Engineering and Physical Sciences Research Council [grant numbers EP/K503058/1 and EP/M010724/1]. 


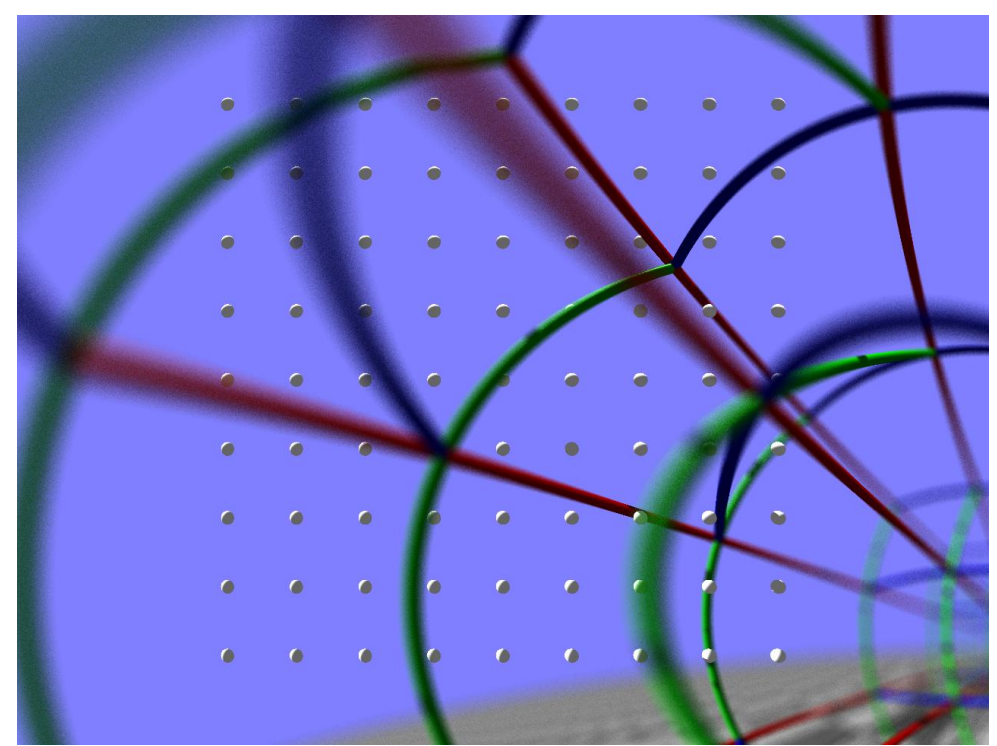

b

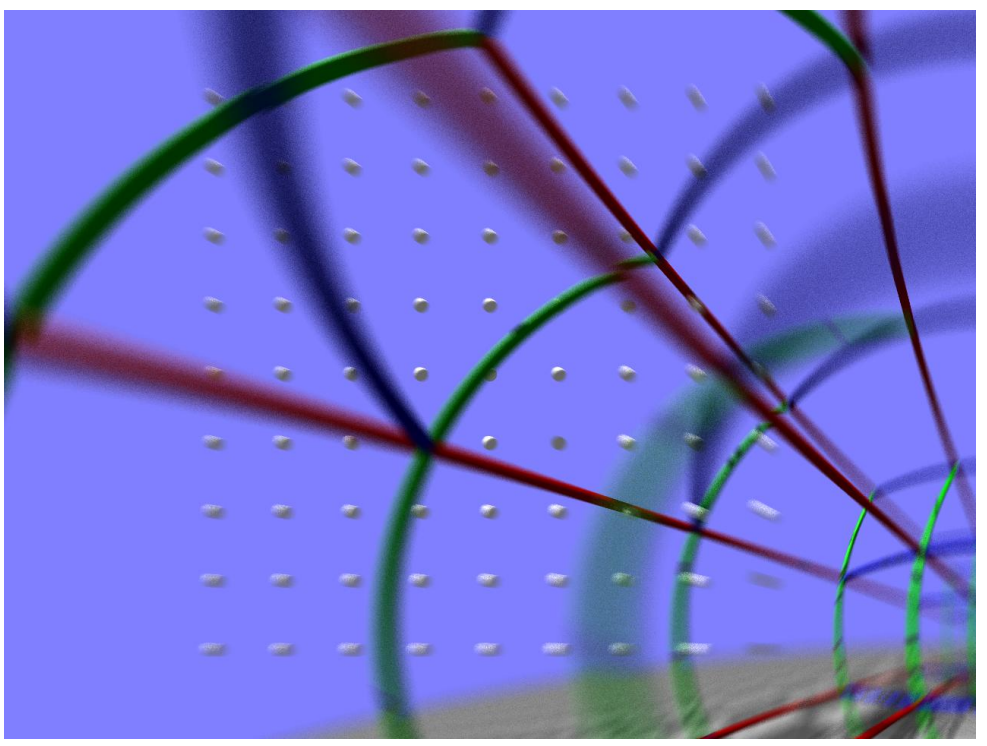

Figure 3. Focussing ability of cameras that use different shutter models. In both simulated photos, the camera attempts to focus on the array of white spheres. Whereas the camera that uses the detector-plane shutter model produces a sharp image of the array of spheres (a), the camera that uses the aperture-plane shutter model does not (b). In contrast to the previous figures, in which cameras with a pinhole aperture were simulated, the cameras use a wide aperture. The figures were calculated using ShutterModelFocussing class in Dr TIM's optics.raytrace.research.RelativisticPhotography package, which can be found in the openly available source code. ${ }^{15}$

\section{REFERENCES}

[1] G. F. FitzGerald, "The Ether and the Earth's Atmosphere," Science 13, p. 390, 1889.

[2] A. A. Michelson and E. Morley, "On the relative motion of the earth and the luminiferous ether," American Journal of Science 34(203), pp. 333-345, 1887.

[3] A. Einstein, "Zur Elektrodynamik bewegter Körper," Annalen der Physik 17, pp. 891-921, 1905.

[4] A. Lampa, "Wie erscheint nach der Relativitätstheorie ein bewegter Stab einem ruhenden Beobachter?," Zeitschrift für Physik 27, pp. 138-148, 1924.

[5] R. Penrose, "The apparent shape of a relativistically moving sphere," Proc. Cambridge Philos. Soc. 55, pp. 137-139, 1959. 


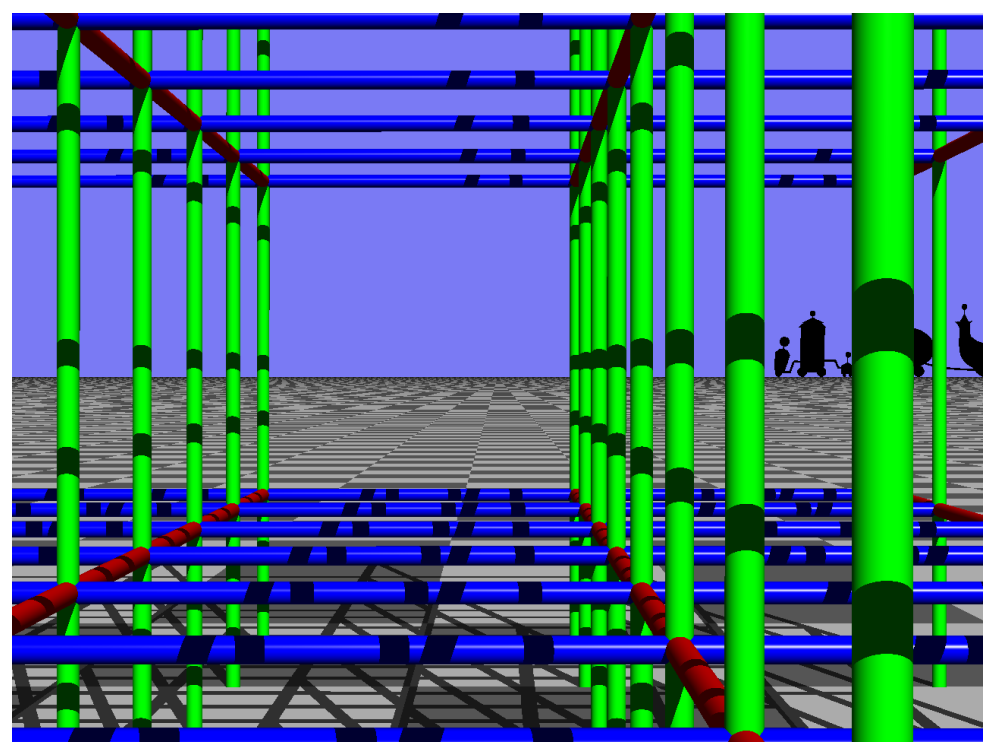

Figure 4. Correction of the relativistic aberration by placing a suitable Lorentz window in front of the lens of a camera that uses the aperture-plane shutter model. The figure was calculated using ShutterModelFocussing class in Dr TIM's optics.raytrace.research. RelativisticPhotography package, which can be found in the openly available source code. $^{15}$

[6] J. Terrell, "Invisibility of the Lorentz contraction," Phys. Rev. 116, pp. 1041-1045, 1959.

[7] M. L. Boas, "Apparent shape of large objects at relativistic speeds," Am. J. Phys. 29, p. 283, 1961.

[8] A. Howard, S. Dance, and L. Kitchen, "Relativistic ray-tracing: Simulating the visual appearance of rapidly moving objects," tech. rep., 1995.

[9] D. Weiskopf, M. Borchers, T. Ertl, M. Falk, O. Fechtig, R. Frank, F. Grave, A. King, U. Kraus, T. Müller, H.-P. Nollert, I. R. Mendez, H. Ruder, T. Schafhitzel, S. Schär, C. Zahn, and M. Zatloukal, "Explanatory and illustrative visualization of special and general relativity," IEEE Transactions on Visualization and Computer Graphics 12, pp. 522-534, 2006.

[10] C. M. Savage, A. Searle, and L. McCalman, "Real time relativity: Exploratory learning of special relativity," Am. J. Phys. 75, pp. 791-798, 2007.

[11] D. Lambert, A. C. Hamilton, G. Constable, H. Snehanshu, S. Talati, and J. Courtial, "TIM, a ray-tracing program for METATOY research and its dissemination," Comp. Phys. Commun. 183, pp. 711-732, 2012.

[12] A. C. Hamilton and J. Courtial, "Generalized refraction using lenslet arrays," J. Opt. A: Pure Appl. Opt. 11, p. 065502, 2009.

[13] S. Oxburgh, T. Tyc, and J. Courtial, "Dr TIM: Ray-tracer TIM, with additional specialist capabilities," Comp. Phys. Commun. 185, pp. 1027-1037, 2014.

[14] S. Oxburgh, N. Gray, M. Hendry, and J. Courtial, "Lorentz-transformation and Galileo-transformation windows," Proc. SPIE 9193, p. 91931K, 2014.

[15] "TIM: A raytracer for forbidden optics." http://sourceforge.net/projects/timray/. 